\title{
Blood Groups and Leprosy
}

\author{
FRANCISCO M. SALZANO
}

\begin{abstract}
From the Departamento de Genética, Instituto de Ciências Naturais, Universidade Federal do Rio Grande do Sul, Pôrto Alegre, Brazil
\end{abstract}

The problem of the relation between blood groups and disease has been the subject of many investigations. An important paper appeared as early as 46 years ago (Buchanan and Higley, 1921). Specific studies about blood groups and leprosy also date back to the twenties (Puente, 1927-28 and others). Since that time the relation between the ABO blood groups and this disease has been tested in different countries and at different times. But no one has ever tried to analyse all these data. The importance of this task will be apparent by an inspection of Table I. Not less than 11,261 patients and 390,602 controls have been studied in 14 different countries. An examination of the 27 series listed shows that the only more or less consistent finding is an apparent excess of $O$ and lack of $\mathrm{B}$ among the leprosy patients as compared with healthy persons. This possible relation was tested by Woolf's (1955) method. The results are listed in the last two columns of Table I. Despite the fact that the incidence of the disease in $\mathrm{O}$ as compared to B persons is greater than 1 in 16, the deviations are not large and in only two Japanese series do they reach the significance level. The combined analysis of all series shows a total relative $\mathrm{O}: \mathrm{B}$ incidence negligibly different from unity. The $\chi^{2}$ for heterogeneity among the series is on the borderline of significance $\left(\chi^{2}=38.3 ; 26\right.$ d.f.; $\mathrm{p}>0.05)$. An analysis was also tried comparing the incidence of the disease among $\mathrm{O}$ and non- $\mathrm{O}$ individuals. But here again the relative incidence is practically equal to unity $(1.02)$ and the $\chi^{2}$ for heterogeneity shows a significant value $\left(\chi^{2}=55 \cdot 8\right.$; 26 d.f. ; $p<0.001$ ).

Table II shows results in relation to the $R h$ system. No clear tendency towards an excess or lack of $\mathrm{Rh}$ negatives among patients is apparent from the data. Accordingly, no further analysis was considered necessary.

Received October 17, 1966.
Table III presents the distribution of $\mathrm{ABO}$ phenotypes among the different forms of the disease. There is a tendency for a lack of $O$ and excess of $B$ among the lepromatous patients, but the differences are small, and only in the series from Ghana are they statistically significant. The over-all $\mathrm{O}: \mathrm{B}$ incidence is $1 \cdot 15$, giving a $\chi^{2}$ of 2.9 for the difference between this value and unity $(p>0.05)$. The $\chi^{2}$ for heterogeneity is 12.9 (7 d.f.; $p>0.05$ ). Beiguelman $(1963,1964)$ has found a significant excess of O persons among tuberculoid patients living in São Paulo, Brazil. This difference, however, decreases when his total series is separated into Whites and Negroids. When the incidence of the different forms of the disease in $\mathrm{O}$ against all other phenotypes in the series listed here is considered, again the only significant value is given by the Ghana patients. The test for heterogeneity in this analysis is significant at the $2 \%$ level if this series is considered. If it is ignored, no significant results are obtained considering all the other series together.

Table IV presents the results of $\mathrm{Rh}$ blood typing in patients with different forms of leprosy. As happened with the $\mathrm{Rh}$ comparison between leprosy patients and normals, no clear tendency is apparent.

There is no indication, therefore, of any differential susceptibility to leprosy or its forms among the carriers of different $\mathrm{ABO}$ and $\mathrm{Rh}$ phenotypes. In relation to the $\mathrm{ABO}$ system the data are sufficiently numerous to rule out any important contribution of genes in this system to the variance in this attribute. Information concerning other systems is still too scarce and need not be considered here.

This work has been supported in part by the Rockefeller Foundation, Conselho Nacional de Pesquisas and PHS research grant GM-08238, Public Health Service, U.S.A. The help of Dr. M. Kawabe in relation to the Japanese reference materials is gratefully acknowledged. 
TABLE I

ABO BLOOD GROUPS AND LEPROSY

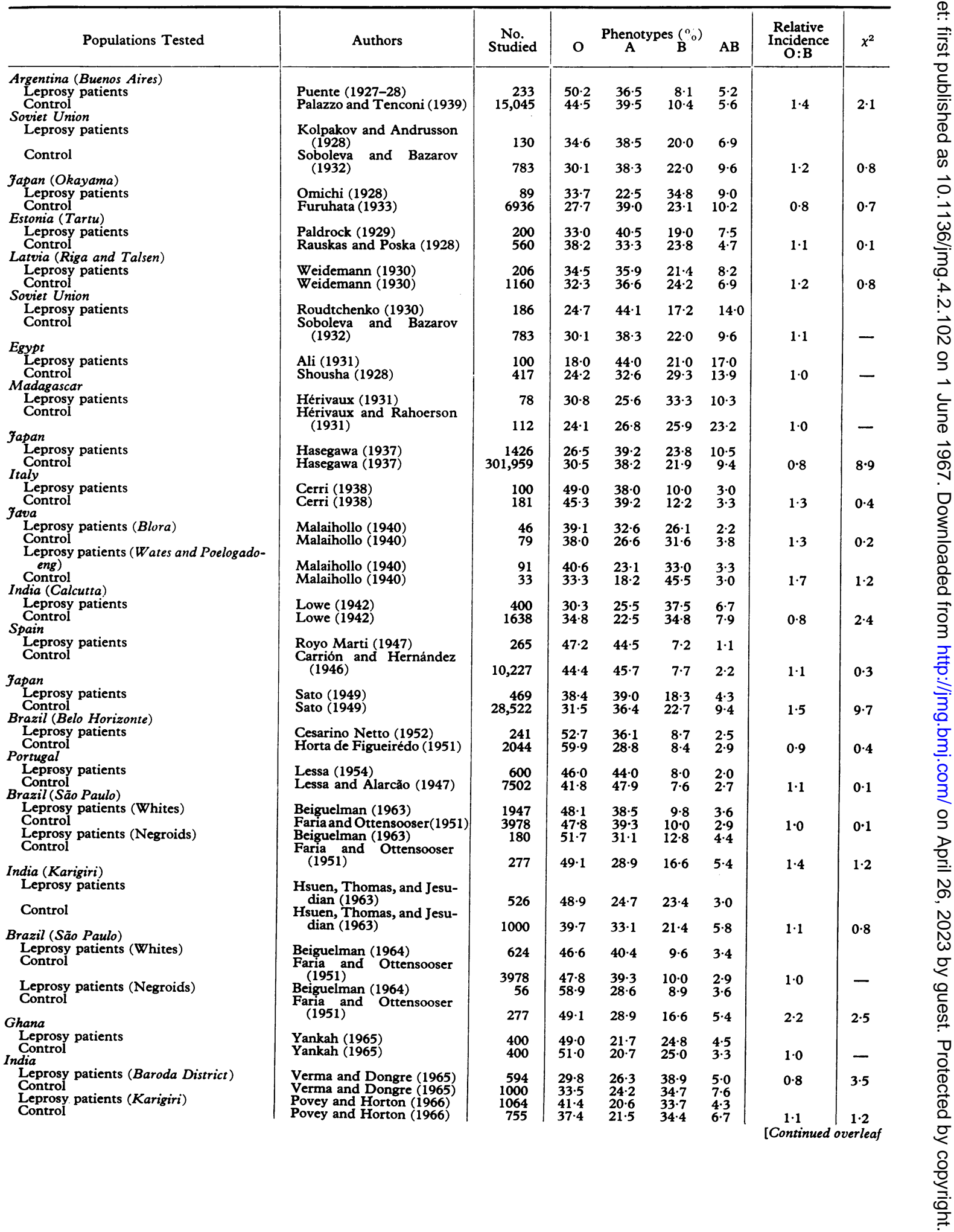


TABLE I (continued)

\begin{tabular}{|c|c|c|c|c|c|c|c|c|}
\hline Populations Tested & Authors & Studied & $\mathbf{O}$ & $\underset{\text { A }}{\text { Phenoty }}$ & ${ }_{B}(\%)$ & $\mathbf{A B}$ & $\begin{array}{c}\text { Relative } \\
\text { Incidence } \\
\mathrm{O}: \mathrm{B}\end{array}$ & $x^{2}$ \\
\hline $\begin{array}{l}\text { Brazil (three cities) } \\
\text { Leprosy patients (Whites) } \\
\text { Control } \\
\text { Leprosy patients (Negroids) } \\
\text { Control }\end{array}$ & $\begin{array}{l}\text { Salzano, Suñé, and Fer- } \\
\text { lauto (1967) } \\
\text { Salzano, Suñé, and Fer- } \\
\text { lauto (1967) } \\
\text { Salzano, Suñé, and Fer- } \\
\text { lauto (1967) } \\
\text { Salzano, Suñé, and Fer- } \\
\text { lauto (1967) }\end{array}$ & $\begin{array}{r}936 \\
572 \\
74 \\
384\end{array}$ & $\begin{array}{l}46 \cdot 3 \\
45 \cdot 8 \\
55 \cdot 4 \\
50 \cdot 3\end{array}$ & $\begin{array}{l}41 \cdot 4 \\
42 \cdot 1 \\
31 \cdot 1 \\
29 \cdot 9\end{array}$ & $\begin{array}{r}9 \cdot 1 \\
8 \cdot 6 \\
13 \cdot 5 \\
16 \cdot 7\end{array}$ & $\begin{array}{l}3 \cdot 2 \\
3 \cdot 5 \\
- \\
3 \cdot 1\end{array}$ & 1.0 & $0 \cdot 1$ \\
\hline
\end{tabular}

Note: The following papers were not included because: (a) Oguma and Kitamura (1931) based their observations in saliva before the relation between the ABO and secretor genes was fully understood; (b) Miyamura (1930) and Pinetti (1931) studied only 12 and 31 cases, respectively; (c) in the communication by $\mathrm{Ri}$-i-sho and Kamikawa (1931) no information is given about the number of people studied; (d) at the time of writing this paper the articles by Hayashi (1929), Malinin and Strukov (1930), Muneichi (1934), Valle (1937), and Markianos,

Karageorgopolou, Mpelezos, and Kouzoutzakoglou (1957) were still not available to me.

When the author did not present a control I have chosen a comparable series studied by a different researcher at about the same time as the leprosy investigation.

TABLE II

Rh BLOOD GROUPS AND LEPROSY

\begin{tabular}{|c|c|c|c|}
\hline Populations Tested & Authors & $\begin{array}{l}\text { No. } \\
\text { Studied }\end{array}$ & $\mathbf{R h}_{(\%)}^{(-)}$ \\
\hline $\begin{array}{l}\text { Brazil (Belo Horizonte) } \\
\text { Leprosy patients } \\
\text { Control } \\
\text { Portugal } \\
\text { Leprosy patients } \\
\text { Control } \\
\text { Brazil (São Paulo) } \\
\text { Leprosy patients } \\
\text { (Whites) } \\
\text { Control } \\
\text { Leprosy patients } \\
\text { (Negroids) } \\
\text { Control } \\
\text { Ghana } \\
\text { Leprosy patients } \\
\text { Control } \\
\text { Brazil (three cities) } \\
\text { Leprosy patients } \\
\text { (Whites) } \\
\text { Control } \\
\text { Leprosy patients } \\
\text { (Negroids) } \\
\text { Control }\end{array}$ & $\begin{array}{l}\text { Cesarino Netto } \\
\text { (1952) } \\
\text { Memória and Bar- } \\
\text { bosa (1957) } \\
\text { Lessa (1957) } \\
\text { " (1948) } \\
\text { Beiguelman (1963) } \\
\text { Da Silva Lacaz } \\
\text { (1951) } \\
\text { Beiguelman (1963) } \\
\text { Faria and Otten- } \\
\text { sooser (1951) } \\
\text { Yankah (1965) } \\
\text { " " " " " } \\
\text { Salzano et al. (1967) } \\
\text { " " " } \\
\text { " } \\
\text { " " }\end{array}$ & $\begin{array}{r}91 \\
316 \\
600 \\
514 \\
\\
1859 \\
3809 \\
180 \\
277 \\
400 \\
400\end{array}$ & $\begin{array}{r}12 \cdot 1 \\
10 \cdot 1 \\
28.8 \\
14.8 \\
\\
9.6 \\
15.4 \\
6.7 \\
13.0 \\
6.7 \\
6.3 \\
\end{array}$ \\
\hline
\end{tabular}

\section{REFERENCES}

Ali, M. (1931). Blood groups among lepers. F. Egypt. med. Ass., $14,119$.

Beiguelman, B. (1963). Grupos sanguíneos e lepra. Rev. bras. Leprol., 31, 34.

- (1964). Sistema ABO e epidemiologia de lepra. Rev. paul. Med., 65, 80.

Buchanan, J. A., and Higley, E. T. (1921). The relationship of blood-groups to disease. Brit. F. exp. Path., 2, 247.

Carrión, J., and Hernández, J. (1946). Cited by Royo Marti (1947).

Cerri, B. (1938). I gruppi sanguigni nella lebbra come eventuale elemento di resistenza. G. ital. Derm., Sif., 79, 791.

Cesarino Netto, J. B. (1952). Grupos sanguíneos na lepra. Arch. mineir. Leproi., 12, 53

Da Silva Lacaz, C. (1951). Novos dados estatísticos sôbre a incidência do fator Rh na cidade de São Paulo (Brasil). Rev. paul. Med., 38, 17.

Faria, R., and Ottensooser, F. (1951). Grupos ABO e tipos de Rh em pretos e mulatos de São Paulo. Arq. Biol. (S. Paulo), 35, 68.

Furuhata, T. (1933). Value of Blood Grouping in Anthropology. Kanazawa University. (Cited by Mourant, Kopeć, and Domaniewska-Sobczak, 1958).
Hasegawa, K. (1937). Ưber die Blutgruppen bei Leprakranken in Japan. Lepro, 8, 59.

Hayashi, F. (1929). Mitsuda's Skin Reaction and Leprosy Classification. The National Leprosarium (Aisei-len).

Hérivaux, A. (1931). Les groupes sanguins dans la lèpre. Bull. Soc. Pathol. exot., 24, 618.

- and Rahoerson, R. (1931). Les groupes sanguins chez les Malgaches de l'Emyrne. Bull. Soc. Path. exot., 24, 247.

Horta de Figueirédo, L. (1951). Resumo sôbre 3.000 classificações de grupos sanguíneos. Arq. Clín. (Rio de f.), 12, 445.

Hsuen, J., Thomas, E., and Jesudian, G. (1963). ABO blood groups and leprosy. Leprosy Rev., 34, 143.

Kolpakov, T., and Andrusson, V. (1928). Lepra und Blutgruppen. Allrussischer Bundeskongress zur Leprabekämpfung. (Summarized in $\mathrm{Zbl}$. Haut-u. Geschl.-Kr., 35, 471, 1930.)

Lessa, A. (1948). O fator Rh em portugueses. F. Méd. (Porto), 11, 29.

(1954). L'Outre-Mer portugais dans la II ${ }^{e m e}$ Exposition Mondiale du Sang. Bol. clin. Hosp. Ultramar (Lisboa), 7, 129.

- (1957). A Individualidade Biológica do Sangue. Livraria Luso-Espanhola, Lisbon. Also (1955) Bol. clin. Hosp. Ultramar (Lisboa), 7, (5), suppl., f. Soc. Ciénc. méd. Lisboa, 3, 1. 
TABLE III

ABO BLOOD GROUPS AND TYPE OF LEPROSY

\begin{tabular}{|c|c|c|c|c|c|c|c|c|}
\hline $\begin{array}{l}\text { Populations Tested and } \\
\text { Type of Leprosy }\end{array}$ & Authors & $\begin{array}{l}\text { No. } \\
\text { Studied }\end{array}$ & $\mathbf{O}$ & $\begin{array}{c}\text { Phenoty } \\
\text { A }\end{array}$ & es ${ }_{\mathbf{B}}^{\left(\begin{array}{l}0 \\
0\end{array}\right)}$ & AB & $\begin{array}{c}\text { Relative } \\
\text { Incidence } \\
\text { O:B }\end{array}$ & $\chi^{2}$ \\
\hline $\begin{array}{l}\text { India (Karigiri) } \\
\text { Lepromatous } \\
\text { Non-lepromatous } \\
\text { Brazil (São Paulo) } \\
\text { Lepromatous (Whites) } \\
\text { Tuberculoid (Whites) } \\
\text { Lepromatous (Negroids) } \\
\text { Tuberculoid (Negroids) } \\
\text { Lepromatous (Whites) } \\
\text { Tuberculoid (Whites) } \\
\text { Ghana } \\
\text { Lepromatous } \\
\text { Tuberculoid } \\
\text { India (Baroda District) } \\
\text { Lepromatous } \\
\text { Non-lepromatous } \\
\text { India (Karigiri) } \\
\text { Lepromatous } \\
\text { Tuberculoid } \\
\text { Dimorphous } \\
\text { Brazil (three cities) } \\
\text { Lepromatous (Whites) } \\
\text { Tuberculoid (Whites) } \\
\text { Indeterminate (Whites) }\end{array}$ & $\begin{array}{l}\text { Hsuen et al. (1963) } \\
\text { Hsuen et al. (1963) } \\
\text { Beiguelman (1963) } \\
\text { Beiguelman (1963) } \\
\text { Beiguelman (1963) } \\
\text { Beiguelman (1963) } \\
\text { Beiguelman (1964) } \\
\text { Beiguelman (1964) } \\
\text { Yankah (1965) } \\
\text { Yankah (1965) } \\
\text { Verma and Dongre (1965) } \\
\text { Verma and Dongre (1965) } \\
\text { Povey and Horton (1966) } \\
\text { Povey and Horton (1966) } \\
\text { Povey and Horton (1966) } \\
\text { Salzano et al. (1967) } \\
\text { Salzano et al. (1967) } \\
\text { Salzano et al. (1967) }\end{array}$ & $\begin{array}{r}1538 \\
409 \\
118 \\
62 \\
312 \\
312 \\
196 \\
204 \\
288 \\
306 \\
382 \\
431 \\
251 \\
721 \\
117 \\
93\end{array}$ & $\begin{array}{l}47 \cdot 3 \\
51 \cdot 3 \\
46 \cdot 6 \\
61 \cdot 3 \\
43 \cdot 3 \\
50 \cdot 0 \\
\\
38 \cdot 3 \\
59 \cdot 3 \\
\\
32 \cdot 0 \\
27 \cdot 8 \\
\\
41 \cdot 6 \\
41 \cdot 5 \\
40 \cdot 6 \\
\\
46.5 \\
43 \cdot 6 \\
48.4\end{array}$ & $\begin{array}{l}27 \cdot 5 \\
16 \cdot 2 \\
25 \cdot 7 \\
26 \cdot 8 \\
\\
19 \cdot 6 \\
20 \cdot 2 \\
22 \cdot 7\end{array}$ & $\begin{array}{r}26 \cdot 4 \\
20 \cdot 5 \\
10 \cdot 1 \\
8 \cdot 6 \\
12 \cdot 7 \\
12 \cdot 9 \\
8 \cdot 0 \\
11 \cdot 2 \\
29 \cdot 6 \\
20 \cdot 1 \\
38 \cdot 5 \\
39 \cdot 2 \\
33 \cdot 8 \\
33 \cdot 7 \\
33 \cdot 9 \\
9 \cdot 4 \\
8 \cdot 5 \\
7 \cdot 5\end{array}$ & $\begin{array}{l}3 \cdot 9 \\
2 \cdot 7 \\
5 \cdot 1 \\
3 \cdot 2 \\
3 \cdot 8 \\
2 \cdot 9 \\
\\
4 \cdot 6 \\
4 \cdot 4 \\
\\
3 \cdot 8 \\
6 \cdot 2 \\
5 \cdot 0 \\
4 \cdot 6 \\
2 \cdot 8 \\
3 \cdot 2 \\
4 \cdot 3 \\
2 \cdot 2\end{array}$ & $\begin{array}{l}1.3 \\
1.3 \\
0.8\end{array}$ & $\begin{array}{l}1.5 \\
0.3 \\
0.4\end{array}$ \\
\hline
\end{tabular}

Data related to old classifications (those utilized before the Havana International Congress of 1948) were not considered.

In the series of Povey and Horton (1966) and Salzano et al. (1967) only the results concerning the lepromatous and tuberculoid types were included in the analysis.

TABLE IV

Rh BLOOD GROUPS AND TYPE OF LEPROSY

\begin{tabular}{|c|c|c|c|c|}
\hline $\begin{array}{l}\text { Populations Tested and } \\
\text { Type of Leprosy }\end{array}$ & \multicolumn{2}{|c|}{ Authors } & $\begin{array}{l}\text { No. } \\
\text { Studied }\end{array}$ & $\mathrm{Rh}\left(-\frac{-}{\%}\right)$ \\
\hline $\begin{array}{l}\text { Brazil (São Paulo) } \\
\text { Lepromatous (Whites) } \\
\text { Tuberculoid (Whites) } \\
\text { Lepromatous (Negroids) } \\
\text { Tuberculoid (Negroids) } \\
\text { Ghana } \\
\text { Lepromatous } \\
\text { Tuberculoid } \\
\text { Brazil (three cities) } \\
\text { Lepromatous (Whites) } \\
\text { Tuberculoid (Whites) } \\
\text { Indeterminate (Whites) }\end{array}$ & $\begin{array}{c}\text { Beiguelman } \\
", \\
\text { " } \\
\text { Yankah (196 } \\
\text { " } " \\
\text { Salzano et al } \\
\text { ", ", }\end{array}$ & $\begin{array}{l}963) \\
\text { ", } \\
\text { ", } \\
(1967) \\
y \\
,\end{array}$ & $\begin{array}{r}1450 \\
409 \\
118 \\
62 \\
196 \\
204\end{array}$ & $\begin{array}{r}9 \cdot 7 \\
9 \cdot 3 \\
5 \cdot 1 \\
9 \cdot 7 \\
6 \cdot 6 \\
6 \cdot 9 \\
14 \cdot 8 \\
9 \cdot 4 \\
10 \cdot 8\end{array}$ \\
\hline
\end{tabular}

—_, and Alarcão, J. (1947). Cited by Mourant, Kopeć, and Domaniewska-Sobczak (1958).

Lowe, J. (1942). Leprosy and blood groups. Leprosy in India, 14, 23.

Malaihollo, J. F. (1940). Lepra in de desa Wates (Res. Batavia) en het resultaat van een bloedgroeponderzoek onder leprozen en gezonden in Wates en Blora. Gen. T. Ned.-Ind., 80, 2296.

Malinin, I., and Strukov, S. (1930). Isoagglutinations-Eigenschaften des Blutes bei Leprakranken. Vrach Delo, 13, 187. (Summarized in Zbl. Haut-u. Geschl.-Kr., 36, 627, 1931.)

Markianos, I., Karageorgopolou, A. I. K., Mpelezos, A., and Kouzoutzakoglou, R. (1957). Scheseis ton homadon haimatos ABO kai paragontos Rhesus epi pashonton ek lepras. Acta microbiol. hellen., 2, 34.

Memória, J. M. P., and Barbosa, M. (1957). Análise estatística dos grupos sanguineos em Belo Horizonte. Arch. Esc. Sup. Vet. Est. Minas Gerais, 10, 299.

Miyamura, K. (1930). UUber die Beziehungen zwischen Hautkrankheiten und Blutgruppen. Fapan F. Dermat., 30, 449 (in Japanese, German summary).

Mourant, A. E., Kopeć, A. C., and Domaniewska-Sobczak, K. (1958). The ABO Blood Groups. Blackwell, Oxford. 3
Muneichi; T. (1934). Beitrage zur Kentnis des Bluttypus bei Leprakranken. fapan f. Dermatol. Urol., 10, 35.

Oguma, H., and Kitamura, T. (1931). On blood types of lepers tested with the saliva. Lepro, 2, 133 (in Japanese, English summary).

Omichi, N. (1928). Die Bedeutung der Blutgruppen im Gebiete der Dermatologie und der Urologie. f. Okayama med. Soc., 40, 1182 (in Japanese, German summary).

Palazzo, R., and Tenconi, J. (1939). Estadistica sobre 15,000 clasificaciones de grupo sanguíneo, realizadas en Buenos Aires. Sem. méd. (B. Aires), 46 (2), 459. (Cited by Mourant, Kopeć, and Domaniewska-Sobczak, 1958).

Paldrock, A. (1929). Die Blutgruppen der Leprösen in Estland. Arch. Schiffs-u. Tropenhyg., 33, 440.

Pinetti, P. (1931). Lo studio del sangue nella lepra. G. ital. Derm. Sif., 72, 1319.

Povey, M. S., and Horton, R. J. (1966). Leprosy and blood groups . Leprosy Rev., 37, 147.

Puente, J. J. (1927-28). Los grupos sanguineos en la lepra. Rev. Asoc. argent. Derm. Sif., 12, 125.

Rauskas, J., and Poska, X. (1928). Cited by Paldrock (1929).

Ri-i-sho, and Kamikawa, Y. (1931). Blood types of lepers in Taiwan. 
4th Meeting, Jap. Ass. for Leprosy, Tokyo. (Summarized in Zbl. Haut-u. Geschl. Kr., 41, 688, 1932.)

Roudtchenko, S. N. (1930). Blood groups in lepers. Trop. Med. Vet. Mosk., 8, 34. (In Russian, with German summary. English summary in Biol. Abstr., 7, 638, 1933.)

Royo Marti, R. (1947). Grupos sanguíneos y lepra. Fontilles (Alicante), 7, 609.

Salzano, F. M., Suñé, Margarete V., and Ferlauto, Marlene (1967). New studies on the relationship between blood groups and leprosy. Acta genet. (Basel). In the press.

Sato, E. (1949). ABO blood groups and secretion especially in relation to familial leprosy. Lepro, 18, 65 (in Japanese).

Shousha, A. T. (1928). On the biochemical race-index of the Egyptians. F. Egypt. med. Ass., 11, 4.

Soboleva, G. V., and Bazarov, N. I. (1932). Antrop. Zh., no. 2, 124. (Cited by Mourant, Kopeć, and Domaniewska-Sobczak, 1958.)

Valle, A. (1937). Déterminations des groups sanguins chez les lepreux de l'Hospice Prophylactique. Rev. med. Hyg. trop., 29, 125.
Verma, B. S., and Dongre, A. V. (1965). Leprosy and ABO blood groups. Leprosy Rev., 36, 211.

Weidemann, M. (1930). Zur Verteilung der Blutgruppen bei den Leprösen Lettlands. Med. Klin., 26, 1155.

Woolf, B. (1955). On estimating the relation between blood group and disease. Ann. hum. Genet., 19, 251.

Yankah, J. A. K. (1965). Observation on the frequency of ABO and $\mathrm{Rh}$ blood groups in leprosy and non-leprosy people in Ghana. Leprosy Rev., 36, 73.

\section{Addendum}

A further study has appeared while this paper was in the press: Gupta, M. C., and Gupta, S. R. (1966). Blood groups in relation to pulmonary tuberculosis and leprosy. Indian F. med Sci., 20, 353. 